\title{
Role of thermodynamics in extensions of mesoscopic dynamical theories
}

\author{
Miroslav Grmela ${ }^{1 *}$ \\ ${ }^{1}$ École Polytechnique de Montréal, \\ Montréal, Québec, Canada \\ *Email address for correspondence: miroslav.grmela@polymtl.ca \\ Communicated by Vito Antonio Cimmelli and David Jou \\ Received on December 12, 2014. Accepted on May 7, 2015.
}

\begin{abstract}
Complex macroscopic systems (like for instance those encountered in nanotechnology and biology) need to be investigated in a family of mesoscopic theories involving varying amount of details. In this paper we formulate a general thermodynamics providing a universal framework for such multiscale viewpoint of mesoscopic dynamics. We then discuss its role in making extensions (i.e. in lifting a mesoscopic theory to a more microscopic level that involves more details).

Keywords: Contact geometry, non-equilibrium thermodynamics, Hamiltonian evolution, dissipation potential, non-local effects.

AMS subject classification: 37K05, 37L05, 37F99.
\end{abstract}

\section{Introduction}

A disagreement between results of experimental observations and theoretical predictions has been the main motivation and the main driving force for extending the classical fluid and continuum mechanics. Mathematical formulations of the extended theories have been then based mainly on mechanics, both macroscopic (i.e. mechanics of continua) and microscopic (i.e. mechanics of microscopic constituents of macroscopic systems under consideration). Our objective in this paper is to discuss the role that thermodynamics has played and should play in such extensions. First, in Section 2, we recall a general formulation of thermodynamics and then, in Section 3, we list some of its contributions to extensions.

Before embarking on this program, we note that there is absolutely no thermodynamics in the initial formulations of the classical fluid mechanics [1] and the classical heat transfer theory. We recall that the classical thermodynamics is indeed younger than these two dynamical theories. Although a version of thermodynamics known as rational thermodynamics [2] 


\section{Thermodynamics in extended dynamics}

was involved in the initial formulations of some of the extensions (see [3]), its role was limited to providing a descriptive framework. We now briefly recall two extensions that are certainly among the oldest.

The first family of important and well established extensions have emerged together with the emergence of plastic (polymer) materials. Fabrication of objects made of plastic materials is done by, first, melting the plastic, and then flowing the melt into molds or extruding it. The flow of polymeric fluids is very different from flows of, say, water, and cannot be predicted by the classical fluid mechanics. Ability to predict flows of plastic materials became in this way directly related to industry and, as a result, investigations (both theoretical and experimental) of extensions of the classical fluid mechanics that are suitable for polymeric fluids received an important financial support and a special name. It became to be known as rheology (the name was coined be Eugene C. Bingham). The theoretical rheology followed two routes. The basis of the first one (see e.g. [4]) was mechanics of various mechanical models of polymeric macromolecules (e.g. a dumbbell model) composing the polymeric fluids. Rational thermodynamics provided the descriptive framework and constituted the basis of the second route (see e.g. [3]).

The second example of the family of well established extensions are the extensions that have arisen in the heat transfer. The classical Fourier theory disagrees with experimental observations in the speed of propagation (the Fourier theory predicts the infinite speed) and in essentially all predictions if the heat transfer is observed in microscale and nanoscale. The macroscopic mechanics (e.g. bringing inertia into heat transfer in [5]) and microscopic mechanics of phonons [6] have led to various extensions known now by the name "phonon hydrodynamics".

\section{Thermodynamics}

Mechanics is sufficient to describe completely the time evolution of macroscopic systems only if the state variables are chosen to be position vectors and velocities of all (i.e. $\sim 10^{23}$ ) particles composing them. If the state variables are less detailed then thermodynamics has to supplement mechanics to formulate the macroscopic dynamics. We now ask and answer three questions.

1. Question: Why do we ignore details? Answer: In order to be able to see a pattern (an overall behavior observed experimentally in macroscopic measurements).

2. Question: How do we know which details are important and which we can afford to ignore? Answer: Since the answer to this question depends 


\section{Grmela}

on both our interests (i.e. on the way we observe experimentally the macroscopic systems under consideration and on intended applications) and on the microscopic dynamics itself (the microscopic time evolution that we can ignore is the fast time evolution that is sufficiently separated from the time evolution of our main interest), we introduce a family of levels of description with varying amount of details. The one with more details we shall call more microscopic (or equivalently less macroscopic). The family represents a multilevel (also called a multiscale) description of macroscopic systems.

3. Question: How do the ignored details influence the time evolution of the details that we still keep in our mathematical formulation? Answer: It is thermodynamics that answers this question. We shall now explain it.

\subsection{Classical equilibrium thermodynamics}

In order to see how thermodynamics deals with ignored details, we recall how the particular case of thermodynamics, namely the classical equilibrium thermodynamics, does it. The setting of the classical equilibrium thermodynamics is limited to static behavior described by the overall volume $V$, the overall energy $E$, and a state variable characterizing the quantity (e.g. the overall number of moles $N$ ). The ignored details are all the remaining information about the microscopic particles composing the macroscopic systems under consideration. In this setting we thus have two levels, namely the microscopic level with all details and the macroscopic level with $(V, E, N)$ playing the role of state variables on which no time evolution takes place.

The microscopic details enter the formulation of the classical equilibrium thermodynamics in two steps. First, the energy $E$ is let to include the so called internal energy that is in fact the energy of all the microscopic particles composing the macroscopic systems. The second step consists of introducing a function $S=S(V, E, N)$, called a fundamental thermodynamic relation. It is in this function where the individual nature of macroscopic systems is expressed. All fundamental thermodynamic relations are however required to share the following properties: (S1): $S$ is a real valued and sufficiently regular function of $(V, E, N),(\mathrm{S} 2): S$ is a concave function, (S3): $S(\lambda V, \lambda E, \lambda N)=\lambda S(V, E, N)$, where $\lambda \in \mathbb{R},(\mathrm{S} 4) \frac{\partial S}{\partial E} \geq 0 ; S=0$ if $\left(\frac{\partial S}{\partial E}\right)^{-1}=0,(\mathrm{~S} 5): S$ tends to its maximum allowed by constraints involving $V, E$, and $N$. It is the influence of ignored details that drives the entropy to its maximum. In the static setting this tendency of the entropy to reach a maximum is the so called Maximum Entropy Principle [7], [8], in the dynamical setting, discussed in the next section in (4), it appears as a result of the time evolution. 


\section{Thermodynamics in extended dynamics}

\subsection{General thermodynamics}

Now we lift the classical equilibrium thermodynamics to a general thermodynamics. We consider two general levels of description: Level $\mathrm{F}$ and Level $G$ that is more macroscopic (i.e. involving less details) than Level F. The time evolution takes place, in general, on both levels. The general thermodynamics that we shall formulate below addresses the question of how the two levels, namely Level F and Level G, are related.

First we introduce a notation. The state spaces used on Level $F$ and Level $\mathrm{G}$ are denoted by $M$ and $N$ respectively, the state variables by $x \in M$, $y \in N$.

The fundamental thermodynamic relation in general thermodynamics is given by

$$
\begin{aligned}
& S: M \rightarrow \mathbb{R} ; x \mapsto S(x) \in \mathbb{R} \\
& Y: M \rightarrow N ; x \mapsto Y(x) \in N
\end{aligned}
$$

The first line in (1) is the same as in the classical equilibrium thermodynamics in Section 2.1 (only the state space has changed). The second line in (1) is new. It is a mapping relating the two levels F and G. Moreover, we assume that the space $N$ has an additional structure: its elements $y \in N$ can always be written as $y=(E, m)$, where $E$ is the energy per unit volume and $m$ are the remaining state variables in the space $N$. Consequently, $Y(x)=(E(x), m(x))$. The fundamental thermodynamic relation in general thermodynamic provides the the entropy $S(x)$ (as it does in the classical equilibrium thermodynamics) and in addition also a mapping relating the two levels and the energy $E(x)$.

The essence of thermodynamics is to maximize the entropy $S(x)$ subjected to constraints [7], [8]. From the mathematical point of view, this type of operations are Legendre transformations. We thus declare that the group of transformations providing the foundation of thermodynamics is the group of Legendre transformations. From this we now follow the standard route (established originally in the elementary particle physics) that will lead us to the geometry of thermodynamics. The question we ask is of what is the geometrical environment in which Legendre transformations are natural transformations. The answer to this question is: it is the contact geometry. This is because the 1-form defining the contact structure is preserved in Legendre transformations. If for instance the fundamental group of transformations were the group of rotations then we would arrive in the same way to metric geometry since the metric tensor is preserved in rotations. It was Hermann in [9] who realized that the contact geometry is the most appropriate geometry for thermodynamics. 


\section{Grmela}

We now introduce the contact geometry in which we formulate general thermodynamics We begin by introducing the space $\mathbf{M}=T^{*} M \times \mathbb{R}$. Its elements are $\left(x, x^{*}, \varphi\right)$. The space $\mathbf{M}$ is naturally equipped with the 1 -form $\theta^{M}=d \varphi-x^{*} d x$ representing the contact structure.

Next, we start to build up a setting for formulating geometrically the fundamental thermodynamic relation (1) and also for the time evolution in which the space $M$ reduces to the space $N$. We introduce a new space $\mathbf{M}^{\mathbf{N}}=T^{*} M \times N^{*} \times N \times \mathbb{R}$ with elements $\left(x, x^{*}, y^{*}, y, \varphi\right), x \in M, x^{*} \in$ $T_{x}^{*} M, y^{*} \in N^{*}, y \in N, \varphi \in \mathbb{R}$. The space $\mathbf{M}^{\mathbf{N}}$ is naturally equipped with the 1-form $\theta^{\left(M^{N}\right)}=d \varphi-x^{*} d x-y^{*} d y$. In $\mathbf{M}^{\mathbf{N}}$ we introduce the thermodynamic potential

$$
\Phi\left(x, y^{*}\right)=-S(x)+<y^{*}, Y(x)>
$$

We shall assume that $S$ and $Y$ are such that $\Phi$ defined in (2) is a convex function of $x$. The manifold representing thermodynamics in $\mathbf{M}^{\mathbf{N}}$ (the thermodynamics specified by the fundamental thermodynamic relation (1)) is now its submanifold

$\mathcal{M}=\left\{\left(x, x^{*}, y^{*}, y, \varphi\right) \in \mathbf{M}^{\mathbf{N}} \mid x^{*}=\Phi_{x}\left(x, y^{*}\right), y=\Phi_{y^{*}}\left(x, y^{*}\right), \varphi=\Phi\left(x, y^{*}\right)\right\} ;$

(3) $\left(x, y^{*}\right) \hookrightarrow\left(x, \Phi_{x}\left(x, y^{*}\right), y^{*}, \Phi_{y^{*}}\left(x, y^{*}\right), \Phi\left(x, y^{*}\right)\right)$

called a Gibbs-Legendre manifold in $\mathbf{M}^{\mathbf{N}}$. We use hereafter the abbreviated notation $\Phi_{x}=\frac{\partial \Phi}{\partial x}$.

We note that in various projections of $\mathcal{M}$ we see geometrically all the elements of the maximization of the entropy $S(x)$ subjected to constraints $Y(x)$. Namely:

(i)

The manifold $[\mathcal{M}]_{y^{*}=0}$ displays the fundamental thermodynamic relation (1). Indeed, the manifold $[\mathcal{M}]_{y^{*}=0}$ is the image of the mapping $x \hookrightarrow\left(x,-S_{x}(x), 0, Y(x),-S(x)\right)$.

(ii)

The manifold $[\mathcal{M}]_{x^{*}=0}$ displays the state $x_{t h}\left(y^{*}\right)$ in $M$ at which $S(x)$ subjected to constraints $Y(x)$ reaches its maximum and also the fundamental thermodynamic relation $S=S(y)$ in $N$ implied by the fundamental thermodynamic relation (1) in $M$. Indeed, the manifold $[\mathcal{M}]_{x^{*}=0}$ is the image of the mapping $\left(x, y^{*}\right) \hookrightarrow\left(x_{t h}\left(y^{*}\right), 0, y^{*}, Y\left(x_{t h}\left(y^{*}\right)\right), S^{*}\left(y^{*}\right)\right)$, where $S^{*}=S^{*}\left(y^{*}\right)$ is the Legendre transformation of the fundamental relation $S=S(y)$ in $N$ that is implied by the fundamental thermodynamic relation $S=S(x)$ in $M$ and by the mapping $Y: M \rightarrow N$.

Now, we turn to the time evolution in which Level $\mathrm{F}$ reduces to Level G. In the static situation that we have discussed so far, the passage 


\section{Thermodynamics in extended dynamics}

$S(x) \rightarrow S(y)$ is made by Legendre transformation (i.e. by maximizing the entropy $S(x)$ subjected to constraints $Y(x))$. We recall that Legendre transformation in $\mathbf{M}^{\mathbf{N}}$ is a transformation preserving its contact structure (i.e. the 1-form $\left.\theta^{\left(M^{N}\right)}=d \varphi-x^{*} d x-y^{*} d y\right)$. Let us now construct a time evolution that makes the Legendre transformation leading from $S(x)$ to $S(y)$. In other words, we look for a time evolution in $\mathbf{M}^{\mathbf{N}}$ that preserves its contact structure and brings $S(x)$ to $S(y)$ as $t \rightarrow \infty$. We shall call such time evolution a thermodynamic time evolution. To find it is our first problem.

The key result that allows us to tackle the first problem is the knowledge of the canonical form (see e.g. [10]) of the time evolution preserving the contact structure. In the particular case of $\mathbf{M}^{\mathbf{N}}$ equipped with the 1-form $\left.\theta^{\left(M^{N}\right)}=d \varphi-x^{*} d x-y^{*} d y\right)$, the time evolution preserving $\theta^{\left(M^{N}\right)}$ is governed by

$$
\begin{aligned}
\dot{x} & =\Psi_{x^{*}} \\
\dot{x^{*}} & =-\Psi_{x}+x^{*} \Psi_{\varphi} \\
\dot{y}^{*} & =\Psi_{y} \\
\dot{y} & =-\Psi_{y^{*}}+y \Psi_{\varphi} \\
\dot{\varphi} & =-\Psi+<x^{*}, \Psi_{x^{*}}>+<y, \Psi_{y}>
\end{aligned}
$$

where

$$
\Psi: \mathbf{M}^{\mathbf{N}} \rightarrow \mathbb{R}
$$

is called a contact Hamiltonian.

The remaining problem is to identify the contact Hamiltonian $\Psi$ satisfying the following three requirements:

$$
\begin{aligned}
& \text { (1) }:[\Psi]_{\mathcal{M}}=0 \\
& (2): \mathcal{M} \text { is an invariant manifold } \\
& (3): \mathcal{M} \rightarrow \mathcal{N}^{*} \text { as } t \rightarrow \infty
\end{aligned}
$$

The first property is a general property of the contact Hamiltonian (see e.g. [11]), the second and the third properties are physical requirements. We want the thermodynamic time evolution to take place on the GibbsLegendre manifold representing the thermodynamics and we want the time evolution to bring the thermodynamics from the more microscopic Level $\mathrm{F}$ to the more macroscopic Level G.

The three properties (6) do not single out the contact Hamiltonian. Below, we present three examples of $\Psi$. 


\section{Grmela}

Quadratic contact Hamiltonian

$$
\Psi\left(x, x^{*}, y^{*}, y, \varphi\right)=\frac{1}{2}\left(<\Phi_{x}, \Lambda \Phi_{x}>-<x^{*}, \Lambda x^{*}>\right)
$$

where $\Lambda: M^{*} \rightarrow M$ is an operator satisfying the following properties

$$
\Lambda \text { is independent of }\left(x, x^{*}, y^{*}, y, \varphi\right)
$$

$\Lambda$ is symmetric and positive definite

$$
\Lambda Y_{x}=0
$$

We now verify the properties (6). The first property is obviously satisfied. In order to investigate the second and the third properties, we evaluate (4) on $\mathcal{M}$. We obtain

$$
\begin{aligned}
\dot{x} & =-\Lambda \Phi_{x} \\
\dot{x^{*}} & =-\Phi_{x x} \Lambda \Phi_{x} \\
\dot{y^{*}} & =0 \\
\dot{y} & =0 \\
\dot{\Phi} & =-<\Phi_{x}, \Lambda \Phi_{x}>
\end{aligned}
$$

We thus indeed see that the manifold $\mathcal{M}$ is invariant and that the thermodynamic potential $\Phi$ plays the role of the Lyapunov function for the approach $x \rightarrow x_{t h}\left(y^{*}\right)$ where $x_{t h}\left(y^{*}\right)$ is a solution of $\Phi_{x}=0$. This proves that $\mathcal{M} \rightarrow \mathcal{N}^{*}$ as $t \rightarrow \infty$.

We also note that from the mathematical point of view, the dynamics (9) is a gradient dynamics. The vector field is generated by gradient of a potential (the potential in our case is $\Phi$ ) that is transformed into a vector by a metric tensor (in our case it is $\Lambda$ ).

\section{Dissipation potential}

The quadratic in $x^{*}$ function $\left\langle x^{*}, \Lambda x^{*}>\right.$ appearing in (7) can be replaced by a more general (convex in $x^{*}$ ) function $\Xi$ called a dissipation potential. We thus arrive at the second example of the contact Hamiltonian:

$$
\Psi\left(x, x^{*}, y^{*}, y, \varphi\right)=\left[\Xi\left(x, x^{*}\right)\right]_{x^{*}=\Phi_{x}}-\Xi\left(x, x^{*}\right)
$$

where the dissipation potential $\Xi: T^{*} M \rightarrow \mathbb{R}$ is required the following 


\section{Thermodynamics in extended dynamics}

properties:

$\Xi$ is independent of $\left(y^{*}, y, \varphi\right)$

$\Xi$, as a function of $x^{*}$ reaches its minimum at $(x, 0)$

$\Xi$ is a convex function $x^{*}$ in a neighborhood of $(x, 0)$

$<Y_{x},[\Xi]_{x^{*}=S_{x}}>=0$

$$
[\Xi]_{x^{*}=Y_{x}}=0
$$

If (4) is restricted to $\mathcal{M}$ we obtain

$$
\begin{aligned}
\dot{x} & =-\left[\Xi_{x^{*}}\right]_{x^{*}=\Phi_{x}} \\
\dot{x^{*}} & =-\Phi_{x x}\left[\Xi_{x^{*}}\right]_{x^{*}=\Phi_{x}} \\
\dot{y}^{*} & =0 \\
\dot{y} & =0 \\
\dot{\Phi} & =-\left[<x^{*}, \Xi_{x^{*}}>\right]_{x^{*}=\Phi_{x}}
\end{aligned}
$$

The proof of the properties (6) follows closely the proof in the previous example. We note that the essence of the extension from (7) to (10) is that the inequality $\xi^{2}>0$, where $\xi \in \mathbb{R}$, can be written as $\xi \Xi_{\xi}>0$, where $\Xi(\xi)=\frac{1}{2} \xi^{2}$ and that the inequality holds also for more general functions $\Xi$, namely for those that share with $\frac{1}{2} \xi^{2}$ the following three properties: (i) $\Xi(0)=0$, (ii) $\Xi$ reaches its minimum at 0 , and (iii), $\Xi$ is a convex function in a neighborhood of 0 .

From the mathematical point of view, the dynamical system (12) is a nonlinear generalization of the gradient dynamical system.

Contact Hamiltonian combining gradient and symplectic dynamics

Having in mind the first property in (6), we note that we can add to (7) or to (10) a term that, if evaluated on $\mathcal{M}$, becomes zero because of an antisymmetric multiplication rather than because of subtraction as it is in (7) or in (10). Such term is obviously $\left\langle x^{*}, L \Phi_{x}\right\rangle$, where $L: M^{*} \rightarrow M$ is a skew-symmetric operator. We thus propose

$$
\Psi\left(x, x^{*}, y^{*}, y, \varphi\right)=-\mathcal{S}\left(x, x^{*}, y^{*}, y, \varphi\right)+\frac{1}{e^{*}} \mathcal{H}\left(x, x^{*}, y^{*}, y, \varphi\right)
$$

where

$$
\mathcal{S}\left(x, x^{*}, y^{*}, y, \varphi\right)=\Xi\left(x, x^{*}\right)-\left[\Xi\left(x, x^{*}\right)\right]_{x^{*}=\Phi_{x}}
$$

and

$$
\mathcal{H}\left(x, x^{*}, y^{*}, y, \varphi\right)=<x^{*}, L \Phi_{x}>
$$




\section{Grmela}

where the dissipation potential $\Xi$ is required to satisfy the properties (11) and the operator $L$ the properties

$L$ is skewsymmeric

$$
L S_{x}=L m_{x}=0
$$

We recall that $y=(E, m)$ and $Y(x)=(E(x), m(x))$, where $E(x)$ is the energy per unit volume and $m(x)$ are the remaining state variables in the space $N$.

Before leaving the contact geometry formulation (4), (13) of the general thermodynamics we make another comment indicating the advantage. We note that with the choice (13) of the contact Hamiltonian the first two equations in (4) can be obtained as Euler-Lagrange equations (variations are made with respect to $x$ and $x^{*}$ ) corresponding to the functional

$$
\mathcal{I}=\int d t\left[\Psi\left(x, x^{*}, y^{*}\right)-<x^{*}, x>\right]
$$

We note that with the choice (13) of the contact Hamiltonian the quantity $[\mathcal{I}]_{\mathcal{M}}$ has the physical interpretation of the total entropy generated during the approach from Level $\mathrm{F}$ to Level $\mathrm{G}$. The variational formulation $\delta \mathcal{I}=0$ of the governing equations of general thermodynamics has thus the following physical interpretation. The level $\mathrm{F}$ approaches the Level $\mathrm{G}$ in such a way that the entropy generated during the approach reaches its extremum. If we recall the many advantages of Hamilton's variational principle in classical mechanics, we can anticipate similar advantages of the variational principle $\delta \mathcal{I}=0$.

Now, we leave the contact geometry formulation in $\mathbf{M}^{\mathbf{N}}$ by restricting ourselves only to its invariant submanifold $\mathcal{M}$. If we evaluate (4) with (13) playing the role of the contact Hamiltonian on $\mathcal{M}$ we arrive at

$$
\begin{aligned}
& \dot{x}=L E_{x}+\left[\Xi_{x^{*}}\right]_{x^{*}=S_{x}} \\
& \dot{S}=\left[<x^{*}, \Xi_{x^{*}}>\right]_{x^{*}=S_{x}}
\end{aligned}
$$

We have omitted the equation $\dot{y}^{*}=0$ and the equations governing the time evolution of the conjugate variables since they are (on $\mathcal{M}$ ) straightforward consequences of (18). The second equation in (18) is also a straightforward consequence of the first equation but we keep it in order to manifestly display the thermodynamic content of the first equation in (18).

When passing from (4) to (18) we seem to be gaining (in apparent simplicity) but we are also loosing. From the mathematical point of view, the dynamics (18) is a combination of a Hamiltonian and gradient dynamics. 


\section{Thermodynamics in extended dynamics}

We recall that in order that $\dot{x}=L E_{x}$ be a Hamiltonian dynamics we need to assume that $L$ satisfies an additional property, namely that $\left\langle A_{x}, L B_{x}\right\rangle$, where $A$ and $B$ are real valued sufficiently regular functions of $x \in M$, is a Poisson bracket (i.e. beside being antisymmetric it also satisfies the Jacobi identity). We note a very important mathematical difference between the contact-structure formulation (4), (13) and the explicit formulation (18). In the geometrical formulation (4), (13) the combination of the two types of dynamical systems is made by using one geometrical structure (namely the contact structure) to transform gradients of two potentials (namely $\mathcal{S}$ and $\mathcal{H}$ ). In the explicit formulation (18), the combination is made by using two geometrical structures (namely the symplectic structure $L$ and the metric structure $\Lambda$ ) to transform gradient of one potential (namely the thermodynamic potential $\Phi$ ). This difference makes the formulation (4), (13) more geometrical than the formulation (18).

Another important difference between (4), (13) and (18) is that the former admits the variational formulation (17) but the latter, to the best of our knowledge, does not.

The contact structure formulation of both equilibrium and nonequilibrium thermodynamics has been first proposed in [12]. More recent formulations with many illustrations can be found in [13], [14]. The explicit formulation (18) (namely the first equation in (18)) has emerged gradually as an abstract equation collecting the essential features of mesoscopic dynamics in [15], [16], [17], [18], and in [19], [20] where it was called GENERIC (an acronym for General Equation for Non-Equilibrium Reversible-Irreversible Coupling).

As for the concept of entropy $S$ that we have introduced in the general thermodynamics, we emphasize that $S(x)$ depends on both the starting Level $\mathrm{F}$ and the target Level $\mathrm{G}$. It is therefore a concept depending on our needs and our intentions (it is, as it is sometimes said, an anthropic concept). In this context it is however important to emphasize that while it is true that we are free to choose the $F$ and $G$ levels (and thus the entropy), it is up to the macroscopic system itself (namely its microscopic time evolution) to determine as to whether the levels that we have chosen are or are not acceptable (i.e. providing a self-contained viewpoint in which results of experimental observations are reproducible and are found to be in agreement with theoretical predictions).

We now recall some particularly important examples of the general thermodynamics.

Level F: Boltzmann kinetic theory; Level G: equilibrium thermodynamics

This is the example that in fact gave birth to the formulation of general 


\section{Grmela}

thermodynamics. It is also historically the first example of a mesoscopic theory for which its relation to the classical equilibrium thermodynamics has been investigated. In this example Level $\mathrm{F}$ is the level of one particle kinetic theory (i.e. $x=f(\boldsymbol{r}, \boldsymbol{v})$ ), the time evolution is governed by the Boltzmann kinetic equation, $S(x)=-k_{B} \int d \boldsymbol{r} \int d \boldsymbol{v} f(\boldsymbol{r}, \boldsymbol{v})(\ln f(\boldsymbol{r}, \boldsymbol{v})-1)$, $k_{B}$ is the Boltzmann constant, and the dissipation potential is the potential arising in chemical kinetics (binary collisions are regarded as binary chemical reactions of species labeled by $\boldsymbol{v}$ - see [16], [21]). The mapping $Y$ is in this setting given by $f(\boldsymbol{r}, \boldsymbol{v}) \mapsto\left(\int d \boldsymbol{r} \int d \boldsymbol{v} f(\boldsymbol{r}, \boldsymbol{v}), \int d \boldsymbol{r} \int d \boldsymbol{v} \frac{v^{2}}{2 m} f(\boldsymbol{r}, \boldsymbol{v})\right)$.

Gibbs equilibrium statistical mechanics; Level F classical mechanics of particles; Level G: equilibrium thermodynamics

Level $\mathrm{F}$ in this example is the level of $N \sim 10^{23}$ particle mechanics. Macroscopic systems are seen as composed of atoms and molecules. In the particle representation, $x$ are the position coordinates and momenta of all particles. In the Liouville representation, $x$ is N-particle distribution function $f_{N}$, where $\mathrm{N}$ is the number of particles in the macroscopic system. In the Gibbs equilibrium statistical mechanics, the entropy is chosen to be given by a universal expression $S(x)=-k_{B} \int f_{n} \ln f_{N}$, where $\int$ denotes integration over the phase space of all $N$ particles. The energy $E(x)$ is the Hamiltonian of $N$ particles composing the macroscopic system. It is thus in $E(x)$ where the individual nature of the macroscopic systems under considerations is expressed. The time evolution equation of the type (18) is replaced in the Gibbs equilibrium statistical mechanics by: (i) two most important consequences of the classical mechanics of particles, namely the conservation of the total mass and the total energy, and (ii) by an assumption about particle trajectories, namely that the particle trajectories are such that the averages made by following the trajectories (i.e. averages in time) can be replaced by certain type of averages in the phase space.

Level F: classical fluid mechanics; Level G: equilibrium thermodynamics

In this example, level $\mathrm{F}$ is the level of classical fluid mechanics, $x=$ the five classical hydrodynamic fields, the Poisson bracket is the one found (implicitly) by Clebsch [22] and explicitly by Arnold [11] (see (19) below). The general-thermodynamics formulation of the classical fluid mechanics is presented for example in [13].

Level F: Cattaneo heat conduction theory; Level G: Fourier heat conduction theory

In this example, $x=(e(\boldsymbol{r}), \boldsymbol{w}(\boldsymbol{r}))$, where $e$ is the energy field and $\boldsymbol{w}$ is a vector field related to the heat flux. On Level $\mathrm{G}, x=e(\boldsymbol{r})$. The generalthermodynamics formulation of the approach level $\mathrm{F} \rightarrow$ Level $\mathrm{G}$ has been worked out in [23]. What is particularly interesting in this example is the emerging entropy. It appears to be the classical local equilibrium entropy 
supplemented with a nonlocal Cahn-Hilliard term (see also Section 3.4 below) multiplied by a parameter involving the heat conductivity coefficient.

\section{Role of thermodynamics in extensions}

As we noted in Section 1, extended mesoscopic dynamical theories have been initially introduced on the basis of arguments whose origin lies mainly in mechanics (both microscopic and macroscopic) and, in lesser extent, in rational thermodynamics. Other versions of thermodynamics then joined the process of creating extensions in [24] and references cited in [13].

We shall now comment about the role that the general thermodynamics presented in Section 2.2 above plays in extensions. First, we recall that the principal role of a model is to provide a setting that allows to organize, predict and explain results of a certain family of experimental observations. We can divide the observations into two types: quantitative and qualitative. The former are observations whose outcome is a quantitative relation among two or more parameters (for example a graph representing measured shear stress versus imposed shear rate). The latter experimental observations are observations of the compatibility between two levels of description. By this we mean that the behavior of a fluid under investigation is found to be well described on a Level $\mathrm{F}$ and, after some preparation, also on a more macroscopic Level G. For example, behavior of a fluid that is seen to be well described by the classical fluid mechanics is found, if left sufficiently long time without external influences, also well described by the classical equilibrium thermodynamics. We require from the model to agree with both types of observations. Regarding the quantitative experimental observations, we need to solve the governing equations in all details in order to verify the agreement. Qualitative properties of solutions are needed to verify the qualitative experimental observations. As we have seen in Section 2.2, the qualitative properties of solutions expressing the compatibility of two levels are guaranteed if the governing equations possess the structure of general thermodynamics. This then means that if we know from experimental observations that the Levels $\mathrm{F}$ and $\mathrm{G}$ are compatible then the model on Level $\mathrm{F}$ that agrees with this experimental observations has to have the structure of the general thermodynamics. Below, we list some of the contributions to extensions that the requirement of agreement of qualitative predictions with qualitative experimental observations (i.e. the general thermodynamics formulated in Section 2.2) brings. 


\section{Grmela}

\subsection{Framework}

The first contribution of general thermodynamics is that it provides a framework for constructing extensions. The structure (18) involves modules:(i) state variables, $x$, (ii) their kinematics expressed in the Poisson bracket $\{$,$\} , (iii) dissipation potential, and (iv) the generating potentials$ $(E, S)$ and the mapping $Y$. We formulate extended dynamical theories gradually, module by module. This process is very efficient (see e.g. [25]).

\subsection{Mathematical regularity}

Ideally, the physical regularity should come hand in hand with the mathematical regularity. By the former we mean a clear physical meaning and agreement between model predictions and results of experimental observations, by the latter a clear mathematical formulation in which the questions like existence of solutions and their regularity are addressed. Indeed, the physical processes are seen experimentally to exist so that they should exist also in the mathematical formulation as solutions to the governing equations. This is in fact a basic requirement of agreement between experimental observations and theoretical predictions. In reality however it is rarely the case that the physical clarity implies directly the mathematical clarity. We may recall for example that the Boltzmann kinetic equation, that has served since its introduction at the end of nineteen century as a beacon of physical clarity in mesoscopic dynamics, has only recently been clarified in [26] from the mathematical point of view. It is therefore very interesting to note that at least in some particular settings the structure of general thermodynamics (or sometimes only some of its elements) addresses also the clarity of the mathematical formulation. For example, it is the case when the time evolution equations are a system of local conservation laws ( [27], [28]). In such setting the entropy equation (which in this case is another local conservation law) implies the mathematical regularity (existence and uniqueness of solutions). Even in the case of the Boltzmann equation [26], the proof of the existence of its solutions uses some of the elements of the thermodynamic structure as for instance the entropy equation (in this context it is Boltzmann's H-theorem) and the conjugate form of the Boltzmann equation (the concept of the Boltzmann equation in a renormalized sense introduced in [26] is closely related to the conjugate form of the Boltzmann equation).

\subsection{Fundamental thermodynamic relation, conjugate state variables}

The classical nonequilibrium thermodynamics brings the classical fluid mechanics to a relation with the classical thermodynamics. This modification of the classical fluid mechanics can be seen in fact as historically 


\section{Thermodynamics in extended dynamics}

its first extension. The newly introduced state variable is the entropy field $s(\boldsymbol{r})$. However, contrary to the extensions mentioned in Section 1, this new state variable is not an independent field but a function (satisfying certain properties) of the original hydrodynamic fields. The extension in this case consists in the requirement that the entropy field evolves in time in a certain way. This viewpoint of nonequilibrium thermodynamics and the corresponding to it investigation of its consequences on constitutive relations (i.e. on relations expressing the fluxes that arise on the right hand side of local conservation laws in terms of the hydrodynamic fields) is an important contribution of rational thermodynamics [29]. We shall now show what new to this investigation brings the general-thermodynamics viewpoint of extensions.

First, we prove that in the setting of the classical fluid mechanics without dissipation (i.e. in the setting of the Euler fluid mechanics) the requirement of the extra local conservation law for the entropy field $s(\boldsymbol{r})$ (in addition to the local conservation laws for the mass $\rho(\boldsymbol{r})$, momentum $\boldsymbol{u}(\boldsymbol{r})$, and energy $e(\boldsymbol{r})$ fields), supplemented with the requirement of the Hamiltonian structure, implies the local equilibrium assumption. In other words, the entropy field has to be a specific function of the hydrodynamic fields. It is not only a function satisfying the properties required from all entropy functions (i.e. (i) $s$ is a sufficiently regular function of the hydrodynamic fields, (ii) it is a concave function, (iii) $\frac{\partial s}{\partial e}(\boldsymbol{r}) \geq 0$ ) but it is a specific function whose dependence on the mass, momentum and energy is locally the same as at equilibrium. This result illustrates well the extra power that the requirement of the Hamiltonian structure brings to nonequilibrium thermodynamics.

Now we proceed to the proof.

Let the state variables in the classical fluid mechanics be chosen to be $x=(\rho(\boldsymbol{r}), s(\boldsymbol{r}), \boldsymbol{u}(\boldsymbol{r})$, the fundamental thermodynamic relation is $E=$ $\int e(\boldsymbol{r})$, where $e(\boldsymbol{r})=e(\rho, s, \boldsymbol{u} ; \boldsymbol{r})$. The kinematics of $(\rho(\boldsymbol{r}), s(\boldsymbol{r}), \boldsymbol{u}(\boldsymbol{r})$ is given [11] by the Poisson bracket

$$
\begin{aligned}
\{A, B\}= & \int d \boldsymbol{r}\left[\rho\left(\partial_{i}\left(A_{\rho}\right) B_{u_{i}}-\partial_{i}\left(B_{\rho}\right) A_{u_{i}}\right)\right. \\
& +s\left(\partial_{i}\left(A_{s}\right) B_{u_{i}}-\partial_{i}\left(B_{s}\right) A_{u_{i}}\right) \\
& \left.+u_{j}\left(\partial_{i}\left(A_{u_{j}}\right) B_{u_{i}}-\partial_{i}\left(B_{u_{j}}\right) A_{u_{i}}\right)\right]
\end{aligned}
$$

We use the summation convention. The time evolution equations (18) with- 


\section{Grmela}

out the last term on its right hand side becomes now

$$
\begin{aligned}
\frac{\partial \rho}{\partial t} & =-\partial_{i}\left(\rho E_{u_{i}}\right) \\
\frac{\partial s}{\partial t} & =-\partial_{i}\left(s E_{u_{i}}\right) \\
\frac{\partial u_{i}}{\partial t} & =-\partial_{j}\left(u_{i} E_{u_{j}}\right)-\rho(\boldsymbol{r}) \partial_{i} E_{\rho(\boldsymbol{r})}-s(\boldsymbol{r}) \partial_{i} E_{s(\boldsymbol{r})}-u_{k}(\boldsymbol{r}) \partial_{i} E_{u_{k}(\boldsymbol{r})}
\end{aligned}
$$

We note that the third equation becomes a local conservation law

$$
\frac{\partial u_{i}}{\partial t}=-\partial_{j}\left(u_{i} E_{u_{j}}+\delta_{i j} p(\boldsymbol{r})\right)
$$

(i.e. the the total momentum will be conserved) provided the quantity $p$ is related to the fields $(\rho(\boldsymbol{r}), s(\boldsymbol{r}), \boldsymbol{u}(\boldsymbol{r})$ by

$$
p(\boldsymbol{r})=-e(\boldsymbol{r})+\rho(\boldsymbol{r}) E_{\rho(\boldsymbol{r})}+s(\boldsymbol{r}) E_{s(\boldsymbol{r})}+\boldsymbol{u}(\boldsymbol{r}) E_{\boldsymbol{u}(\boldsymbol{r})}
$$

But this relation is exactly the local equilibrium assumption with $p(\boldsymbol{r})$ playing the role of local pressure. This ends the proof.

Before leaving this section we make two comments about conjugate state variables.

Fluxes depend on conjugate state variables rather than on the variables themselves

An important motivation for constructing extensions of the classical fluid mechanics has been Grad's hierarchy [30]. The Boltzmann kinetic equation governing the one particle distribution function $f(\boldsymbol{r}, \boldsymbol{v})$ is reformulated in Grad's hierarchy into an infinite system of equations governing the time evolution of an infinite number of fields (i.e. quantities depending only on the position vector $\boldsymbol{r}$ ) representing moments (i.e. quantities of the type $\int d \boldsymbol{v} \zeta(\boldsymbol{v}) f(\boldsymbol{r}, \boldsymbol{v})$, where $\zeta$ is a, in general tensorial, function of $\left.\boldsymbol{v}\right)$. The moments are chosen in such a way that the first five of them are the classical hydrodynamic fields. The first five equations in Grad's hierarchy are thus the classical Euler equations but coupled (in expressions for the fluxes) to higher order moments for which we have their own time evolution equations that are however again coupled to still higher moments. The structure arising in Grad's hierarchy has indeed guided extensions introduced in [31], [32], [24], [33]. Unfortunately, the physical limitation of the Boltzmann equation (only the dynamics of an ideal gas is described by this equation) makes the emerging mathematical structure incomplete. Consequently, Grad's hierarchy is not a good guide for making extensions.

The Grad hierarchy is infinite. If we want to consider only a finite number of fields as state variables, the hierarchy has to be closed. This means 


\section{Thermodynamics in extended dynamics}

that the higher order moments have to be expressed in terms of the lower order moments that are kept as state variables. It is the entropy field (a function of the state variables) and the requirement on its time evolution that is then used to identify the closure [34], [32]. But when we introduce the entropy field we are also introducing conjugate fields that are its derivatives. As we have seen in general thermodynamics in Section 2.2, the conjugate fields play an important role in the time evolution. Roughly speaking, the conjugate variables, rather than the variables themselves, appear on the right hand side of the time evolution equations (i.e. in the vector field). But this exactly is not seen in Grad's hierarchy due to the particularity of the ideal gas. The fields arising in Grad's hierarchy as flows on the right hand side are identical (except possibly a constant multiplicative factor) as the fields serving as state variables. This has then led to the following extension recipe: "take a flux and promote it into the status of an independent extra state variables". From the physical point of view this type of extension means an introduction of extra inertia but what is missing is that the quantities arising in the fluxes are not the state variables themselves but rather their conjugates. We recall that we can see this already in the setting of the classical mechanics of particles. Let us begin with $\boldsymbol{r}$, denoting the position vector of a particle, as the state variable. We can arrive at Newton's time evolution equations as follows: We begin with $\dot{\boldsymbol{r}}=\boldsymbol{v}(\boldsymbol{r})$, where $\boldsymbol{v}(\boldsymbol{r})$ is the "flux". Next, we introduce into this equation an inertia. In other words, we extend it. Following the recipe we take the flux and promote it into the status of an independent state variable $\boldsymbol{v}$. But by doing this we do not end up with Newton's equations. We do however succeed if we consider the flux $\boldsymbol{v}$ as a conjugate of another variable, called momentum $\boldsymbol{u}$, that we adopt as a new independent state variable. The second equation governing its time evolution becomes $\dot{\boldsymbol{u}}=F(\boldsymbol{r}, \boldsymbol{u})$, where $F$ is a force.

Different types of arguments leading to the same conclusion have been introduced in the context of the Grad hierarchy in [35] and in the context of continuum mechanics in [36], [37].

Measurability of conjugate state variables

Another interesting point concerning the conjugate state variables is their measurability (see e.g. [38]). In particular, this question is important for the conjugate of energy whose inverse is called temperature.

In the context of classical thermodynamics the measurability of the temperature is very simple, straightforward and universal. This fact makes the temperature a very important, from both practical and theoretical points of view, quantity characterizing systems at equilibrium. We recall the physics that is behind the measurement of temperature. Because of: (i) maximization of entropy allowed by constraints, and (ii) a universal availability of 


\section{Grmela}

the thermodynamic wall that is transparent for the internal energy (i.e. the energy that remains if the energy expressed in terms of the mechanical state variables that we keep in the classical thermodynamics is subtracted) but closed to the changes in the remaining state variables of classical thermodynamics, two systems (one of them is a thermometer) separated by the thermodynamic wall mentioned in the point (ii) have at equilibrium the same temperatures.

Outside of equilibrium and in the setting of mesoscopic descriptions, we still have a clear definition of temperature as an inverse of the conjugate of energy but its measurability (and thus its importance and practical applicability) is completely lost. This is because of the lack of availability of thermodynamic walls that freely pass the energy (or a part of the energy that we have selected) and keep the remaining state variables constant. The standard thermometer with the standard thermodynamic wall is of no use. It is appealing to think still about a general temperature as about a measure of "agitation" in macroscopic systems. Indeed, we can for example hear in conversations that the temperature of society increased due to introduction of austerity measures. There does not seem to be however a way to measure such temperature and thus to give it a universal applicability and importance that it enjoys at equilibrium.

It is, of course, possible to attempt to measure some quantities that reduce at equilibrium to the equilibrium temperature and outside of equilibrium (or local equilibrium) serve as an indication of "agitation". It does not seem however that any of such quantities can get a universal appeal that would be comparable to the appeal that the equilibrium temperature enjoys. As an example we recall some of the temperatures used in numerical simulations. In this setting measuring means extracting an information from the trajectories (calculated with the assistance of computers) of the particles composing the macroscopic system under consideration. Two types of temperatures are usually measured. One is called kinetic temperature and the other configurational temperature. Both address relation of entropy to energy. The entropy is the one emerging in the equilibrium statistical mechanics and the energy is the energy of the particles (kinetic in the case of the kinetic temperature and potential in the case of the configurational temperature). The problem here is, of course, in the definition of entropy. As we are trying to emphasize in this paper, the entropy depends on the levels of description of our interest and also on how far from equilibrium we are. For example, neither the kinetic nor the configurational temperature is expected to be a good measure of "agitation" in systems with a very anisotropic distribution of velocities. 


\subsection{Nonlocal extensions (Cahn-Hilliard)}

The classical fluid mechanics and the classical continuum theory have been developed for systems that can be inhomogeneous but only moderately. Fluids with large inhomogeneities, as for instance fluids that are near gas-liquid transition, have to be investigated with an extended theory (see e.g. [39], [40], [41], [42], [43] ). We make two remarks about the role of general thermodynamics in this type of extensions.

\section{Dynamical consequences of the Cahn-Hilliard terms in gener- ating potentials}

Following Cahn and Hilliard [44], we extend the classical fluid mechanics by letting the generating potentials (i.e. the energy and the entropy) depend nonlocally on the hydrodynamic fields. In the first approximation we consider only a special nonlocal dependence (called a weak nonlocal dependence). We let the generating potentials at the position $\boldsymbol{r}$ depend on the hydrodynamic fields at $\boldsymbol{r}$ and also on their gradients with respect to $\boldsymbol{r}$ at the position $\boldsymbol{r}$. The question is then of what are the consequences of the new terms involving the gradients (called Cahn-Hilliard terms) in the time evolution equations. The formulation of general thermodynamics is particulary suitable for answering this question. This is because the formulation (4) is, from the mathematical point of view, a weak formulation (note that the Poisson bracket, or alternatively the rate-of-energy potential $\mathcal{E}$, and the rate-of-entropy potential $\mathcal{S}$ involve integrals over $\boldsymbol{r}$ ) and it involves functional derivatives. If the potentials depend, following Cahn and Hilliard, also on gradients then the functional derivatives turn simply into variational derivatives. Otherwise, the mathematical formulation remains unchanged. In this way we can see immediately that, for example, inclusion of a term involving gradient of mass density in the energy potential makes the originally (i.e. in the absence of the Cahn-Hilliard term in the energy) inelastic fluid into an elastic fluid (see details in [40]).

\section{Origin of the Cahn-Hilliard terms in generating potentials}

We can ask the question of what is the physical origin of the CahnHilliard terms in the energy and the entropy potentials. First, following Cahn and Hilliard (see also [43]), it is the presence of large spatial inhomogeneities or alternatively also the presence of long range interactions for which the interaction energy is a nonlocal function of state variables. The general thermodynamics shows however still another route to Cahn-Hilliard terms.

As we have seen in Section 2.2, the general thermodynamics provides the passage: Level $\mathrm{F} \rightarrow$ Level G. If Level $\mathrm{F}$ is the level of the classical fluid mechanics and Level $\mathrm{G}$ is the level of the classical thermodynamics then 


\section{Grmela}

the physics behind the presence of Cahn-Hilliard terms in the generating potentials on Level $\mathrm{F}$ is the physics mentioned in the previous paragraph. If however we consider Level $\mathrm{F} \rightarrow$ Level $\mathrm{G}$ with Level $\mathrm{G}$ being the level of the classical fluid mechanics and Level $\mathrm{F}$ a more microscopic level (e.g. the level of the one particle kinetic theory) then the generating potentials, if evaluated on the target Level G, arise naturally with the Cahn-Hilliard terms as a consequence of the presence of gradients in the Navier-StokesFourier dissipative part of the time evolution on Level G. This has been worked out in Chapter 4 of [13] and in the particular context of heat transfer in [45], see also in [23].

Having shown that one of the origins of the presence of nonlocal terms in the entropy is their presence in the dissipative part of the time evolution, we can ask the question of how do they arise there. In the context of Grad's hierarchy, this question is investigated in [46].

\subsection{Mass-type fields and energy-type fields}

The

fields

serving

as state variables in Grad's hierarchy can be naturally divided into two families, one mass-type and the other energy-type. The mass-type are the moments $\left(\int d \boldsymbol{v} f(\boldsymbol{r}, \boldsymbol{v}), \int d \boldsymbol{v} \boldsymbol{v} f(\boldsymbol{r}, \boldsymbol{v}), \ldots, \int d \boldsymbol{v} \zeta_{i_{1}, \ldots, i_{n}}^{(n)}(\boldsymbol{v}) f(\boldsymbol{r}, \boldsymbol{v}), \ldots\right)$, and the energy-type fields the moments $\left(\int d \boldsymbol{v} v^{2} f(\boldsymbol{r}, \boldsymbol{v}), \int d \boldsymbol{v} v^{2} \boldsymbol{v} f(\boldsymbol{r}, \boldsymbol{v}), \ldots\right.$,

$\left.\int d \boldsymbol{v} v^{2} \zeta_{i_{1}, \ldots, i_{n}}^{(n)}(\boldsymbol{v}) f(\boldsymbol{r}, \boldsymbol{v}), \ldots\right)$, where $\zeta_{i_{1}, \ldots, i_{n}}^{(n)}$ is a polynomial function of $\boldsymbol{v}$ of order $n$. The first two terms in the sequence of mass-type fields are mass and mass flux, the first two terms in the energy-type fields are energy and energy flux. This extra mathematical structure in the state variables of Grad's hierarchy has been noted and used (it seems independently) by three different groups of authors in three different contexts.

First, in [47], the structure has been used to transform the Cattaneo heat transfer theory to Lagrangian coordinates. The fields serving as state variables in the Cattaneo theory consist of two energy-type fields, namely the fields of energy and energy flux, and two mass-type fields, namely the fields of mass and momentum. The pair consisting of mass and momentum fields provides a setting for the classical Lagrangian viewpoint of the fluid flow (as motion of a material fluid particle) and the other pair consisting of the energy and the energy flux fields provides a setting for a new Lagrangian viewpoint of the heat flow (as motion of a "caloric" fluid particle). Altogether, the Cattaneo theory is in this way transformed into a motion of two types of particles, one material and the other caloric. The motion of the two particles is coupled one to the other.

The division of the fields into mass-type and energy-type has been noted 


\section{Thermodynamics in extended dynamics}

and employed in [48].

Finally, the presence of mass-type and energy-type fields in extended hydrodynamic theories has been physically interpreted in thermomass theory [49].

\subsection{Examples of other extensions}

The physics behind the extensions inspired by Grad's hierarchy is an addition of extra inertia into dynamics. There are, of course, also other physical insights motivating extensions. We have already mentioned in Section 1 that among the first extensions were those motivated by internal structure of the fluids (e.g. the presence of long polymer macromolecules or suspended particles). The extra fields in this type of extensions describe the internal (microscopic) structure and the equations governing their time evolution are based on their microscopic physics (see e.g. [4]). The general thermodynamics has been used to investigate this type of extensions in (see references cited in [13]).

Another example of an extension that has its own motivation and its own physical interpretation has been introduced in [50] and independently in [51]. In this extension it is the temperature (i.e a scalar field) that is taken as an extra state variable (i.e. the energy field $e(\boldsymbol{r})$ and the temperature field $T(\boldsymbol{r})$ become in the extended theory two independent scalar state variables). The general-thermodynamics viewpoint of this extension has been discussed in Section VI of [45].

Still another example of extension is physically motivated by an attempt (see [52]) to extend dynamics by including fluctuations. We shall briefly describe it since it is one of the oldest, if not the oldest, extension of classical mesoscopic theories. The essential idea is to extend a deterministic dynamics that is, if experimentally observed, seen to fluctuate to another deterministic dynamics that, if experimentally observed, will not fluctuate or will fluctuate less intensively. The passage from dynamics to its Onsager-Machlup extension is made in five steps: Step 1: The state variables used in the original dynamical theory are promoted to the status of random variables, Step 2: The (deterministic) equation governing the time evolution in the original dynamical theory is upgraded to the Langevintype stochastic equation (i.e. a noise is added to the vector field), Step 3: The resulting stochastic equation is transformed into a Fokker-Planck equation (i.e. the state variable becomes the distribution function), Step 4: The Fokker-Planck equation is formally solved in the form of a functional integral, Step 5: The main contribution to the functional integral arises in the form of Hamilton's variational principle which then implies the extended 


\section{Grmela}

(deterministic) time evolution equations (for details see [52]). To illustrate the Onsager-Machlup extension, we begin with the deterministic equation $\dot{x}=-\lambda \Phi_{x}$, where $x \in \mathbb{R}, \lambda>0, \Phi: \mathbb{R} \rightarrow \mathbb{R}$. After making the five steps we end up with Hamilton's equations $\dot{x}=H_{y} ; \dot{y}=-H_{x}$, with the Hamiltonian $H(x, y)=\lambda\left(\left(\frac{y}{2}\right)^{2}-y \Phi_{x}\right)$, where $y \in \mathbb{R}$ is the extra state variable.

\section{Concluding remarks}

We distinguish between two types of experimental observations: proper (or quantitative), and meta (or qualitative). The outcome of the former is a quantitative relation between two or more types of specific measurements, the outcome of the latter is a qualitative statement about the compatibility of two or more frameworks that have emerged in attempts to make sense of the proper measurements. The qualitative observations are observations about quantitative observations (in other words, meta observations). From the theoretical point of view, the quantitative experimental observations lead to (proper) theories (like e.g. particle mechanics and fluid mechanics), the qualitative experimental observations lead to a theory of theories (i.e. a meta theory). In this paper we argue that such a meta theory is general thermodynamics. Regarding its pertinence, we note that the more complex are the macroscopic system under investigation (as e.g. systems encountered in biology) the less likely it is that their theories can be formulated as single level proper theories and consequently the more pertinent is the general thermodynamics addressing the compatibility relations in the multilevel viewpoint. In particular, we have investigated in this paper the role that the general thermodynamics plays in lifting a given well established mesoscopic theory to a more microscopic level, i.e. to a level involving more details.

\section{Acknowledgements}

The research reported in this paper has been partially supported by Natural Sciences and Engineering Research Council of Canada. I would like to thank also the participants of the special session $\hat{A}$ "Constitutive Equations for Heat Conduction in Nanosystems and Non-equilibrium Processes Â" organized by Vito Antonio Cimmelli and David Jou within the First Joint International Meeting RSME-SCM-SEMA-SIMAI-UMI for very interesting discussions. In particular, the discussion that took place during the closing session gave rise to this paper. 


\section{Thermodynamics in extended dynamics}

\section{REFERENCES}

1. L. Euler, Principes généraux du mouvement des fluides, Académie Royale des Sciences et des Belles-Lettres de Berlin, Mémoires., vol. 11, 1755 (English translation available in Physica D 237, 1825-1839, 2008).

2. C. Truesdell, Rational thermodynamics. New York, NY, USA: Springer, 2nd ed., 1984.

3. R. B. Bird, O. Hassager, R. C. Armstrong, and C. F. Curtiss, Dynamics of Polymeric Fluids, vol. 1. New York, NY, USA: Wiley, 1987.

4. R. B. Bird, O. Hassager, R. C. Armstrong, and C. F. Curtiss, Dynamics of Polymeric Fluids, vol. 2. New York, NY, USA: Wiley, 1987.

5. C. Cattaneo, Sulla conduzione del calore, Atti del Seminario Matematico e Fisico della Universita di Modena, vol. 3, pp. 83-101, 1948.

6. R. Peierls, Zur kinetischen Theorie der Wärmeleitung in Kristallen, Ann. Phys., vol. 395, pp. 1055-1101, 1929.

7. J. W. Gibbs, Collected Works. New York, NY, USA: Longmans Green and Co., 1984.

8. E. T. Jaynes, Foundations of probability theory and statistical mechanics, in Delaware Seminar in the Foundation of Physics, New York, NY, USA: Springer, 1967.

9. R. Hermann, Geometry, Physics and Systems. New York, NY, USA: Marcel Dekker, 1984.

10. V. I. Arnold, Mathematical methods of classical mechanics. New York, NY, USA: Springer, 1989.

11. V. I. Arnold, Sur la géometrie différentielle des groupes de lie de dimension infini et ses applications dans l'hydrodynamique des fluides parfaits, ann, Inst. Fourier, vol. 16, p. 319, 1966.

12. M. Grmela, Thermodynamical lift of the nonlinear onsager-casimir vector field, in Proceedings of the Workshop on Hamiltonian Systems, Transformation Groups and Spectral Transform Methods (J. E. Harnad, J. Marsden, ed.), pp. 199-207, CRM, Univ. de Montreal, 1990.

13. M. Grmela, Multiscale equilibrium and nonequilibrium thermodynamics in chemical engineering, Advances in Chemical Engineering, vol. 39, pp. 76-128, 2010.

14. M. Grmela, Contact geometry of mesoscopic thermodynamics and dynamics, Entropy, vol. 16, pp. 1652-1686, 2014.

15. I. E. Dzyaloshinskii and G. E. Volovick, Poisson brackets in condense matter physics, Ann. Phys., vol. 125, pp. 67-97, 1980.

16. M. Grmela, Particle and bracket formulations of kinetic equations, Contemporary Math., vol. 28, pp. 125-132, 1984. 


\section{Grmela}

17. A. N. Kaufman, Dissipative hamiltonian systems: A unifying principle, Phys. Letters A, vol. 100, p. 419, 1984.

18. P. J. Morrison, Bracket formulation for irreversible classical fields, Phys. Letters A, vol. 100, p. 423, 1984.

19. M. M. Grmela and H. Öttinger, Dynamics and thermodynamics of complex fluids: General formulation, Phys.Rev.E, vol. 56, pp. 6620-6633, 1997.

20. H. C. Öttinger and M. Grmela, Dynamics and thermodynamics of complex fluids: Illustration of the general formalism, Phys.Rev.E, vol. 56, pp. 6633-6650, 1997.

21. M. Grmela, Fluctuations in extended mass-action-law dynamics, Physica D, vol. 241, pp. 976-986, 2012.

22. A. Clebsch, Über die integration der hydrodynamische gleichungen, $J$. Reine Angew. Math, vol. 56, pp. 1-10, 1895.

23. M. Grmela, Role of thermodynamics in multiscale physics, Computers and Math. Appl., vol. 65, pp. 1457-1470, 2013.

24. D. Jou, J. Casas-Vàzquez, and G. Lebon, Extended Irreversible Thermodynamics. Berlin: Springer, 4th ed., 2010.

25. M. Grmela, A. Ammar, and F. C. G. Maîtrejean, A mesoscopic rheological model of moderately concentrated colloids, J. Non-Newtonian Fluid Mech., vol. 212, pp. 1-12, 2014.

26. R. J. DiPerna and P. L. Lions, Global solutions of Boltzmann's equation and the entropy inequality, Arch. Rational Mech. Anal., vol. 114, pp. 47$55,1991$.

27. S. K. Godunov, Symmetric form of the magnetohydrodynamic equation, Chislennye Metody Mekhaniki Sploshnoi Sredy, vol. 3, no. 1, pp. 26-34, 1972.

28. K. O. Friedrichs, Conservation equations and the laws of motion in classical physics, Commun. Pure Appl. Math., vol. 31, pp. 123 - 131, 1978.

29. B. D. Coleman and W. Noll, The thermodynamics of elastic materials with heat conduction and viscosity, Arch. Ration. Mech. Anal., vol. 13, pp. 167-178, 1963.

30. H. Grad, Principles of Kinetic Theory of Gases, vol. 12. SpringerVerlag: Encyclopedia of Physics, 1958.

31. R. T. Müller, I., Rational Extended Thermodynamics. New York, NY, USA: Springer, 1998.

32. T. Ruggeri, The entropy principle: from continuum mechanics to hyperbolic systems of balance laws, Bollettino dell'Unione Matematica Italiana, vol. Serie 8, Vol. 8-B, no. 1, pp. 1-20, 2005. 


\section{Thermodynamics in extended dynamics}

33. V. A. Cimmelli, D. Jou, T. Ruggeri, and P. Ván, Entropy principle and recent results in non-equilibrium theories, Entropy, vol. 16, pp. 17561807, 2014.

34. H. Struchtrup, Macroscopic transport equations for rarefied gas flows. Berlin, Germany: Springer, 2005.

35. G. M. Kremer, An introduction to the Boltzmann equation and transport processes in gases. Berlin, Germany: Springer, 2010.

36. P. Ván, A. Berezovski, and J. Engelbrecht, Internal variables and dynamic degrees of freedom, J. Non-Equilib. Thermod., vol. 33, pp. 235$254,2008$.

37. A. Berezovski, J. Engelbrecht, and M. A. Maugin, Generalized thermomechanics with dual internal variables, Archives Appl. Mech., vol. 81, pp. 229-240, 2011.

38. J. Casas-Vázquez and D. Jou, Temperature in non-equilibrium states: A review of open problems and current proposals, Rep. Prog. Phys., vol. 66, pp. 1937-2023, 2003.

39. M. Grmela, Weakly nonlocal hydrodynamics, Phys. Rev. E, vol. 47, p. 351, 1993.

40. M. Grmela, Extensions of classical hydrodynamics, J. Stat. Phys., vol. 132, pp. 581-602, 2008.

41. V. A. Cimmelli, An extension of liu procedure in weakly nonlocal thermodynamics, J. Mat. Phys., vol. 48, p. 113510, 2007.

42. P. Ván, Weakly nonlocal irreversible thermodynamics, Ann. Physik, vol. 12, pp. 142-169, 2003.

43. E. Johannessen and D. Bedeaux, The nonequilibrium van der waals square gradient model. (ii) local equilibrium of the Gibbs surface, Physica A, vol. 330, pp. 354-372, 2003.

44. J. W. Cahn and J. E. Hilliard, Free energy of a nonuniform system. interfacial free energy, J. Chem. Phys., vol. 28, p. 258, 1958.

45. M. Grmela, G. Lebon, and C. Dubois, Multiscale thermodynamics and mechanics of heat, Phys. Rev. E, vol. 83, 2011.

46. T. Ruggeri, Can constitutive relations be represented by non-local equations?, Quart. Appl. Math., vol. 70, pp. 597-611, 2012.

47. M. Grmela and J. Teichmann, Lagrangian formulation of the MaxwellCattaneo hydrodynamics, Int. J. Engng Sci., vol. 21, pp. 297-313, 1983.

48. T. Arima, S. Taniguchi, T. Ruggeri, and M. Sugiyama, Extended thermodynamics of dense gases, Cont. Mech. Thermodyn., vol. 24, pp. 271$292,2012$.

49. Z. Y. Guo and G. W. Hou, Thermal wawe based on thermomass model, Journal of Heat Transfer, vol. 132, p. 072403, 2010. 


\section{Grmela}

50. V. A. Cimmelli and W. Kosinski, Nonequilibrium semiempirical temperature in materials with thermal relaxation, Arch. Mech., vol. 43, p. 753, 1991.

51. B. L. Holian and P. M. Mareschal, Heat-flow equation motivated by the ideal-gas shock wave, Phys. Rev. E, vol. 82, p. 026707, 2010.

52. L. Onsager and S. Machlup, Fluctuations and irreversible processes, Phys Rev., vol. 91, pp. 1505-1512, 1953. 